\title{
FIGO Stage IVA
}

National Cancer Institute

\section{Source}

National Cancer Institute. FlGO Stage IVA. NCI Thesaurus. Code C96262.

A FIGO stage term that applies to gynecologic cancers. For cervical cancer, it refers to cancer that invades the mucosa of bladder or rectum, and/or extends beyond the true pelvis; for endometrial cancer, it refers to cancer that invades the bladder mucosa and/or the bowel mucosa. 